Reporte de caso / Case Report

http://dx.doi.org/10.18004/mem.iics/1812-9528/2021.019.03.92

\title{
Colitis ulcerosa en paciente con enfermedad celiaca silente.
}

\author{
Raúl Real-Delor ${ }^{(1)}{ }^{1}$, Alba Aveiro ${ }^{(D)}{ }^{1}$, Dora Montiel ${ }^{(1)}{ }^{1}$ Elvira Miskinich ${ }^{1}$ \\ Alexis Benítez ${ }^{(\mathbb{D})}$, Araceli Centurión ${ }^{(i D}$
}

${ }^{1}$ Universidad Nacional de Itapúa. Facultad de Medicina. Postgrado en Medicina Interna. Encarnación, Paraguay

Cómo referenciar este artículo/

How to reference this article:
Real-Delor R, Aveiro A, Montiel D, Miskinich E, Benítez A, Centurión A. Colitis ulcerosa en paciente con enfermedad celiaca silente. Mem. Inst. Investig. Cienc. Salud. 2021; 19(3): 92-95

\section{RE S U M E N}

Se presenta caso de mujer con enfermedad celiaca que presentó colitis ulcerosa complicada con megacolon tóxico. El cuadro revirtió con inmunosupresores y tratamiento médico. Se sugiere a los médicos la búsqueda activa de enfermedades autoinmunes asociadas, además del diagnóstico precoz y tratamiento oportuno de la enfermedad celiaca para evitar complicaciones.

Palabras clave: enfermedad celiaca, enfermedad inflamatoria intestinal, colitis ulcerosa, megacolon tóxico.

\section{Ulcerative colitis in a patient with silent celiac disease.}

\begin{abstract}
A B S T R A C T
We present case of a woman with celiac disease who presented ulcerative colitis complicated with toxic megacolon. The condition reversed with immunosuppressants and medical treatment. Physicians are advised to actively search for associated autoimmune diseases, as well as early diagnosis and timely treatment of celiac disease to avoid complications.
\end{abstract}

Keywords: celiac disease, inflammatory bowel disease, ulcerative colitis, toxic megacolon.

\section{Colite ulcerativa em paciente com doença celíaca silenciosa.}

\section{R E S U M O}

Apresentamos o caso de uma mulher com doença celíaca que apresentou colite ulcerosa complicada com megacolo tóxico. A condição foi revertida com imunossupressores e tratamento médico. Os médicos são aconselhados a pesquisar ativamente as doenças autoimunes associadas, além do diagnóstico precoce e do tratamento oportuno da doença celíaca para evitar complicações.

Palavras-chave: doença celíaca, doença inflamatória intestinal, colite ulcerativa, megacolo tóxico.

\section{INTRODUCCIÓN}

La enfermedad celiaca (EC) es una afección autoinmune, sistémica, originada por la ingesta de gluten en la dieta de personas genéticamente predispuestas. Puede asociarse a otras enfermedades autoinmunes. La colitis ulcerosa es una manifestación 
de la enfermedad inflamatoria intestinal (EII) que puede tener complicaciones agudas y crónicas graves ${ }^{(1)}$.

\section{CASO CLINICO}

Mujer de 17 años acudió al Hospital Nacional (Itauguá, Paraguay) por dolor abdominal de 6 días de evolución. Se acompañó de fiebre graduada en $39{ }^{\circ} \mathrm{C}$ y diarreas sin gleras, pus ni sangre. Fue tratada con cefixima y antipiréticos, sin mejoría. Al cuadro se agregaron vómitos, por lo que acudió a la consulta. La paciente negó sintomatología digestiva previa. Refirió poseer familiares consanguíneos con enfermedad celiaca.

Ingresó vigil, con facies dolorosa, con presión arterial $100 / 70 \mathrm{~mm} \mathrm{Hg}$, pulso $135 / \mathrm{min}$, respiraciones $24 / \mathrm{min}$, temperatura $39{ }^{\circ} \mathrm{C}$, IMC $21,4 \mathrm{k} / \mathrm{m}^{2}$. Al examen físico llamó la atención la palidez de piel y mucosas y el dolor abdominal a la palpación superficial y profunda en epigastrio y flanco derecho, sin irritación peritoneal, con ruidos hidroaéreos presentes. No se detectó visceromegalia.

Se plantearon los diagnósticos sindromáticos de fiebre, dolor abdominal y diarrea aguda. El laboratorio informó hemoglobina $10,5 \mathrm{~g} / \mathrm{dL}$, leucocitos $7460 / \mathrm{mm}^{3}$, neutrófilos $70 \%$, granulaciones tóxicas $5 \%$, sodio $132 \mathrm{mEq} / \mathrm{L}$, potasio $2,7 \mathrm{mEq} / \mathrm{L}$, proteica C reactiva $25 \mathrm{mg} / \mathrm{L}$ (normal $<5 \mathrm{mg} / \mathrm{L}$ ), eritrosedimentación $32 \mathrm{~mm} / 1^{\circ}$ hora, urea $15 \mathrm{mg} / \mathrm{dL}$, creatinina $0,4 \mathrm{mg} / \mathrm{dL}$, albúmina $2,7 \mathrm{mg} / \mathrm{dL}$, procalcitonina $0,66 \mathrm{ng} / \mathrm{mL}$ (normal $<0,5 \mathrm{ng} / \mathrm{mL}$ ). La orina no tenía sedimento patológico y en el frotis de heces había 15 leucocitos/campo y 3 hematíes/campo. El cirujano descartó a priori un cuadro abdominal de resorte quirúrgico. Mientras era hidratada, se corregía su trastorno electrolítico y recibía imipenem y vancomicina llegaron más resultados: anticuerpo IgA antitransglutaminasa $200 \mathrm{mg} / \mathrm{dL}$ (normal <10), anticuerpo IgA antiendomisio (+) 1:80, perfil tiroideo normal, VIH negativo, antígenos febriles para Salmonella negativos, rotavirus negativo, anticuerpos para lupus negativos, hepatitis $\mathrm{B}$ y $\mathrm{C}$ negativos. Los hemocultivos, urocultivo y coprocultivos retornaron negativos.

Fue sometida a endoscopía digestiva alta donde se detectó mucosa duodenal con aspecto en empedrado y pliegues disminuidos en número. Las biopsias informaron cambios compatibles con enfermedad celiaca MARSH 3B/3C (Figura 1).

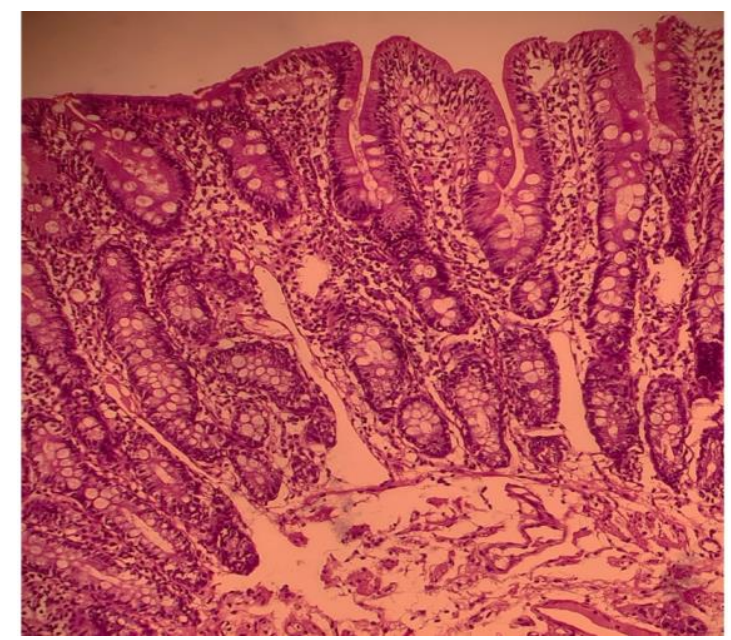

Figura 1. Biopsia duodenal con atrofia de vellosidades, hiperplasia criptal y linfocitosis intraepitelial (>30 linfocitos /100 enterocitos), compatible con enfermedad celiaca Marsh 2B/3C (foto cortesía del Dr. Hugo Boggino).

Ante la persistencia de la fiebre, dilatación del colon (Figura 2) y la aparición de rectorragia es sometida a colonoscopía que detectó colon descendente con ulceraciones lineales y ovaladas, profundas, recubiertas de fibrina, distribuidas en forma parcheada, rodeadas de mucosa edematosa y de fácil sangrado. El recto superior con mucosa congestiva, con realce del patrón vascular. Las biopsias informan cambios compatibles con enfermedad inflamatoria intestinal (Figura 3). 


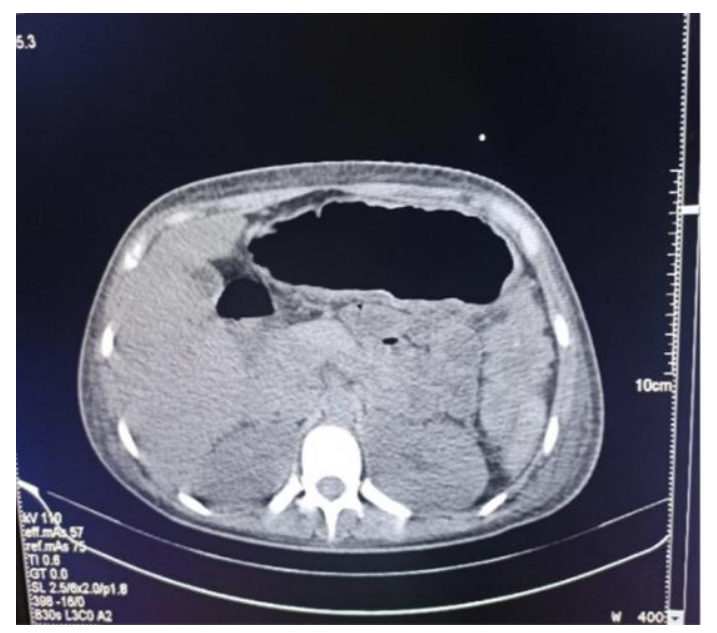

Figura 2. Tomografía simple de abdomen donde se observa dilatación severa del colon transverso.

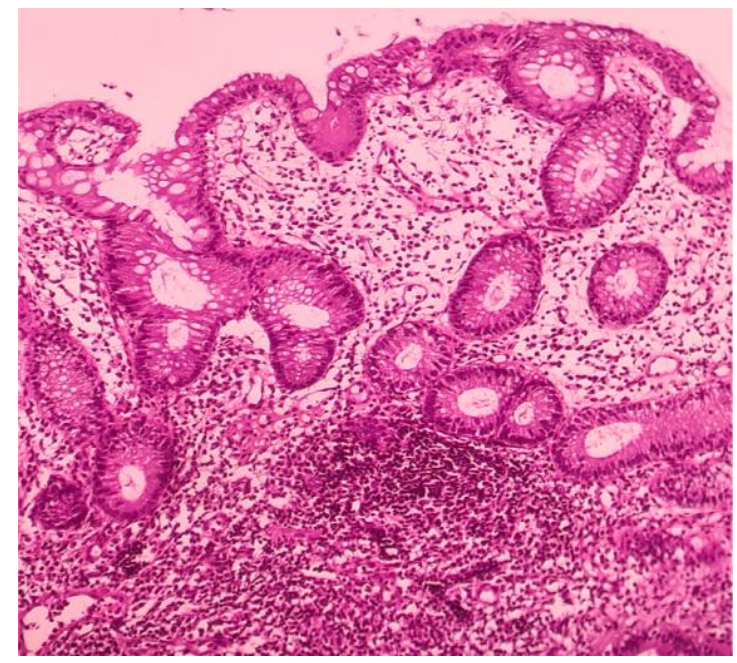

Figura 3. Biopsia de recto-sigmoides con distorsión de la arquitectura glandular, depleción mucinosa leve a moderada, incremento de infiltrado mononuclear en lámina propia y plasmocitosis basal, compatible con enfermedad inflamatoria intestinal (foto cortesía del Dr. Hugo Boggino).

Se inició tratamiento con metilprednisolona intravenosa, alimentación parenteral, se espectó tratamiento quirúrgico, lográndose desaparición de la fiebre, mejoría de los reactantes de fase aguda y del dolor abdominal. La paciente fue de alta con sulfasalazina vía oral, prednisona y dieta sin gluten.

\section{DISCUSIÓN}

La EC de esta paciente se hallaba silente al momento de la consulta, pero fue confirmada con la detección de los autoanticuerpos y la biopsia duodenal (2). En el Paraguay, sólo un tercio de los portadores de EC padecen diarreas crónicas al momento del diagnóstico y $17,6 \%$ tiene familiares consanguíneos con esta patología (3). Es por ello que los médicos deben realizar la búsqueda activa de la EC, sobre todo cuando existen parientes afectos.

La asociación de enfermedades autoinmunes es frecuente ${ }^{(4)}$. En este caso, la asociación entre EC y EII se halla descrita en muchos pacientes ${ }^{(5-7)}$. Lo llamativo de este cuadro es que dominaron los síntomas de la EII, lo que permitió detectar la EC subyacente. Se desconoce si la detección más temprana de la EC y la adecuada dieta sin gluten hubieran evitado la aparición de la EII. 
La presentación clínica de la colitis ulcerosa es variable. El megacolon tóxico pertenece a las complicaciones agudas graves de esta enfermedad, se observa entre 1,6 a $21,4 \%$ de los pacientes con colitis ulcerosa ${ }^{(8)}$. Las principales características del megacolon tóxico son la toxemia, la sepsis y la distensión del colon por disminución del tono muscular, pérdida de la actividad motora y aumento de la cantidad de gas colónico, como se observó en nuestra paciente. Otros investigadores también reportaron el megacolon tóxico como la primera manifestación de la EII ${ }^{(9)}$.

El megacolon tóxico que padeció esta paciente fue sospechado por la presencia de dilatación colónica por la radiografía y tomografía, los signos de toxicidad sistémica y la diselectrolitemia. Se asumió como complicación de la colitis ulcerosa, aunque no pudo descartarse la presencia de $\mathrm{Cl}$. difficile ${ }^{(10)}$. Pero retrospectivamente, fueron los corticoides y la nutrición parenteral los que permitieron la reversión del megacolon tóxico, sin llegar a presentar complicaciones que ameriten cirugía ${ }^{(9)}$.

Se recomienda a los médicos realizar precozmente el diagnóstico de EC y estar atentos a las enfermedades autoinmunes solapadas a esta afección.

\section{Conflicto de intereses}

Los autores declaran no tener ningún conflicto de intereses.

\section{Fuente de financiación}

No se contó con ninguna financiación externa. Se utilizaron recursos propios aportados por los investigadores.

\section{Contribución de autores}

Todos los autores han contribuido conjuntamente y en partes iguales en la elaboración del presente manuscrito.

\section{REFERENCIAS BIBLIOGRÁFICAS}

1. Lebwohl B, Rubio-Tapia A. Epidemiology, presentation, and diagnosis of celiac disease. Gastroenterology. 2021; 160(1): 6375. Doi: https://doi.org/10.1053/j.gastro.2020.0 $\underline{6.098}$

2. Husby S, Murray J, Katzka D. AGA clinical practice update on diagnosis and monitoring of celiac disease: changing utility of serology and histologic measures: Expert review. Gastroenterology. 2019; 156 (4): 8859. Doi: 10.1053/j.gastro.2018.12.010

3. Real Delor R, Arza G, Chamorro M, Dalles I, Ibarra Douglas A. La Enfermedad celiaca en el Paraguay. Rev Nac. 2011; 3(2):7-15. http://scielo.iics.una.py/scielo.php?scrip $\mathrm{t}=\mathrm{sci}$ arttext\&pid=S207281742011000200002

4. Wang L, Wang FS, Gershwin ME. Human autoimmune diseases: $A$ comprehensive update. J Intern Med. 2015; 278(4): 369-95. Doi: 10.1111/joim.12395

5. Manceñido Marcos N, Pajares Villarroya R, Salinas Moreno S, Arribas López MR, Comas Redondo C. Asociación entre enfermedad inflamatoria intestinal de novo y enfermedad celiaca. Rev Esp Enferm Dig. 2020; 112(1): 7-11. Doi: 10.17235/reed.2019.5535/2018
6. Bosca-Watts MM, Minguez M, Planelles D, Navarro S, Rodriguez A, Santiago J, et al. HLA-DQ: Celiac disease vs inflammatory bowel disease. World J Gastroenterol. 2018; 24(1): 96-103. Doi: $10.3748 /$ wjg.v24.i1.96

7. Aziz I, Branchi F, Pearson K, Priest J, Sanders DS. A study evaluating the bidirectional relationship between inflammatory bowel disease and selfreported non-celiac gluten sensitivity. Inflamm Bowel Dis. 2015; 21(4): 84753.

Doi:

10.1097/MIB.0000000000000335

8. Pizarro Jofré G, Quera R, Figueroa C. Factores pronósticos en colitis ulcerosa de reciente diagnóstico. Rev Med Chile. 2017; 145(10): 1319-29. Doi: http://dx.doi.org/10.4067/S003498872017001001319

9. Autenrieth DM, Baumgart DC. Toxic megacolon. Inflamm Bowel Dis. 2012; 18(3): 584-91. Doi: 10.1002/ibd.21847

10. Doshi R, Desai J, Shah Y, Decter D, Doshi S. Incidence, features, in-hospital outcomes and predictors of in-hospital mortality associated with toxic megacolon hospitalizations in the United States. Intern Emerg Med. 2018; 13(6): 881-7. Doi: 10.1007/s11739-018$\underline{1889-8}$ 